\title{
Assessment of Gas-Surface Interaction Models for Computation of Rarefied Hypersonic Flow
}

\author{
Jose F. Padilla* and Iain D. Boyd $\ddagger$ \\ University of Michigan, \\ Ann Arbor, Michigan 48109 \\ DOI: $\underline{10.2514 / 1.36375}$
}

\begin{abstract}
Among the few classes of computational approaches for examining rarefied gas dynamics, the most widely used technique for spatial scales relevant to suborbital spaceflight is the direct simulation Monte Carlo method. One area in which the direct simulation Monte Carlo method can be improved is the numerical modeling of the interactions between gas molecules and solid surfaces. Gas-surface interactions are not well understood for rarefied hypersonic conditions, although various models have been developed. The goal of this study is the assessment of gas-surface interaction models in use with the direct simulation Monte Carlo method. Assessment is made of the two most common gas-surface interaction models in use with direct simulation Monte Carlo: the Maxwell model and the Cercignani, Lampis, and Lord model. The assessment is performed by simulations of flat-plate wind-tunnel tests. Boundary-layer profiles are compared with existing wind-tunnel data. At about $90 \%$ accommodation, both models match the wind-tunnel profiles. Parametric studies demonstrate differences between the model predictions of scattering distributions, boundary-layer profiles, and surface-property distributions. The two models offer similar performance for computing flat-plate aerodynamics.
\end{abstract}

\section{Nomenclature}

$a_{Q}=$ accommodation coefficient of molecular property $Q$

$C_{f} \quad=$ skin-friction coefficient $\tau_{w} / q_{\infty}$

$C_{p} \quad=$ pressure coefficient $\left(p_{w}-p_{\infty}\right) / q_{\infty}$

$f \quad=$ velocity distribution function

$h \quad=$ total enthalpy

$I_{0} \quad=$ modified Bessel function of the first kind and zeroth order

$K=$ scattering kernel

$K n \quad=$ global Knudsen number

$K n_{\text {DGL }}=$ density-gradient-length local Knudsen number

$m \quad=$ mass of one molecule

$n \quad=$ number density

n $=$ local normal unit vector of the solid surface

$P, P_{\max }=$ probability, maximum probability

$p=$ pressure

$q_{\infty} \quad=$ dynamic pressure, $0.5 \rho_{\infty} V_{\infty}^{2}$

$R_{G} \quad=$ particular gas constant

$R_{u} \quad=$ universal gas constant

St $=$ Stanton number, (heat flux at the solid surface divided by $\left.q_{\infty} V_{\infty}\right)$

$T=$ temperature

$T^{*}=$ characteristic temperature of intermolecular potential

$T_{\mathrm{VHS}}=$ reference temperature for the variable-hard-sphere collision model

$\mathbf{t}=$ local resultant tangent unit vector of the solid surface $\left[\left(\mathbf{t}_{1}+\mathbf{t}_{2}\right) /\left|\mathbf{t}_{1}+\mathbf{t}_{2}\right|, \mathbf{t}_{1}\right.$ and $\mathbf{t}_{2}$ are orthonormal $]$

$V \quad=$ magnitude of velocity $|\mathbf{V}|$

Presented as Paper 3891 at the 39th AIAA Thermophysics Conference, Miami, FL, 25-28 June 2007; received 27 December 2007; revision received 9 June 2008; accepted for publication 13 July 2008. Copyright @ 2008 by the American Institute of Aeronautics and Astronautics, Inc. All rights reserved. Copies of this paper may be made for personal or internal use, on condition that the copier pay the $\$ 10.00$ per-copy fee to the Copyright Clearance Center, Inc., 222 Rosewood Drive, Danvers, MA 01923; include the code 0887-8722/ $09 \$ 10.00$ in correspondence with the CCC.

*Graduate Student, Department of Aerospace Engineering. Student Member AIAA.

${ }^{\dagger}$ Professor, Department of Aerospace Engineering. Associate Fellow AIAA.
V

velocity

$W_{p} \quad=$ reference particle weight, $n / n_{\text {simulation particles }}$

$x, y=$ computational domain coordinates relative to the flat-plate leading edge

$Z_{\mathrm{rot}, \infty}=$ maximum rotational collision number

$\alpha=$ energy accommodation coefficient

$\alpha_{\mathrm{vsS}}=$ deflection angle exponent of variable-soft-sphere collision model

$\delta=$ Dirac delta function

$\delta_{\text {diameter }}=$ reference collision cross-sectional diameter

$\zeta=$ number of internal energy degrees of freedom

$\theta_{\text {vib }}=$ characteristic temperature of vibration

$\lambda=$ mean free path

$\mu \quad=$ absolute viscosity

$\xi=$ absolute molecular velocity, $\boldsymbol{\xi}=\mathbf{V}+\boldsymbol{\xi}^{\prime}$

$\boldsymbol{\xi}^{\prime} \quad=$ random molecular velocity

$\rho \quad=$ mass density

$\sigma \quad=$ momentum accommodation coefficient

$\Phi \quad=$ flux

$\omega=$ viscosity index for the variable-hard-sphere collision model

\section{Subscripts}

$E_{\text {int }} \quad=$ internal energy

$e^{=}=$at the edge of the boundary layer

$i \quad=$ incident

$M \quad=$ Maxwell

$\mathrm{mp} \quad=$ most probable

$n=$ relative to surface normal vector

$Q \quad=$ physical property

$r \quad=$ reflected

rot $\quad=$ rotational internal energy mode

$t=$ relative to resultant surface tangent vector $\mathbf{t}$

$t 1, t 2=$ relative to surface tangent vector $\mathbf{t}_{1}$ or $\mathbf{t}_{2}$, respectively

vib $\quad=$ vibrational internal energy mode

$w \quad=$ of the solid-surface wall

$x, y=x$ or $y$ component of the computational domain coordinate

$0=$ reservoir conditions

$\infty \quad=$ freestream or asymptotic value 


\section{Introduction}

$\mathbf{O}$ VER the next few decades, spaceflight is expected to become more common through the resurgence of manned space exploration and the rise of commercial manned spaceflight. An essential role for the efficient research and development of suborbital spaceflight is played by computational simulation of rarefied hypersonic flows. This is because most of these spaceflight endeavors will involve traversing the altitude of $100 \mathrm{~km}$. This is an internationally accepted boundary above which spaceflight begins and is known as the Kármán line [1,2]. It represents the altitude above which sustained aerodynamic lift requires a velocity greater than orbital velocity. Near and above the Kármán altitude is where most rarefied hypersonic flow appears in spaceflight. The flow is rarefied because the associated air density is so low that the flow can no longer be considered a continuum. The flow is hypersonic because the vehicle speed is many times the ambient characteristic speed of sound.

For the rarefied flow conditions of interest, the analysis must account for gas-phase intermolecular collisions to correctly predict the respective aerodynamics and stability. Among the few classes of computational approaches for examining rarefied gas dynamics, the most widely used approach for spatial scales relevant to suborbital spaceflight is the direct simulation Monte Carlo (DSMC) method [3]. Although the DSMC method has been under development for over 40 years, there are still many areas in which improvements can be made [4]. One particular area is the associated numerical modeling of interactions between gas molecules and solid surfaces. Gas-surface interactions are not well understood for rarefied hypersonic conditions, although various models have been developed. This study assesses two common gas-surface interaction models in use with the DSMC method.

A general cell-based implementation of the DSMC method called MONACO [5] is used to scrutinize two of the most common gassurface interaction models implemented into the DSMC method: the Maxwell model, [6] and the Cercignani, Lampis, and Lord (CLL) model [7]. In the literature, comparisons of flowfield properties between DSMC and laboratory data of rarefied hypersonic flow are uncommon. Hence, this study is also intended to generate new comparisons of this kind. To begin the study, a review of gas-surface interactions models is presented. Then a mathematical description of the two common interaction models is laid out. This is followed by a description of the wind-tunnel-test simulations. Afterward, the parametric simulation results are presented and discussed. First, the flow-speed contours and streamlines are examined. Second, probability distribution plots of molecular velocity and surface scattering angle are presented. Third, boundary-layer velocity profiles are compared with existing wind-tunnel data near the leading edge of a flat plate. Comparisons with wind-tunnel boundary-layer profiles lead to an estimate for the gas-surface accommodation coefficient. Fourth, the effects of gas-surface modeling on the surface properties are analyzed. Finally, the results and conclusions are summarized.

\section{Developments in Gas-Surface Interaction Modeling}

Historically, the first gas-surface interaction model for kinetic theory was developed by Maxwell [6] in 1879. It considers two kinds of interactions, the specular and diffuse interactions, which are the result of a molecule encountering a perfectly reflecting or a perfectly accommodating surface, respectively. A specular interaction or reflection occurs when an incident molecule collides with the molecular structure of a solid surface in such a way that it rebounds elastically as if hitting a flat surface. This type of collision occurs when the gas molecule collides with a peak of the solid-surface molecular structure, assuming that the gas and solid molecules are rigid elastic spheres. The collision results in an inversion of the surface normal component of the molecule's incident velocity and no change in its tangential components. Thus, the angle of reflection is the same as the angle of incidence. A diffuse interaction occurs when an incident molecule interacts with the molecular structure of the solid surface in such a way that it attains thermal equilibrium with the

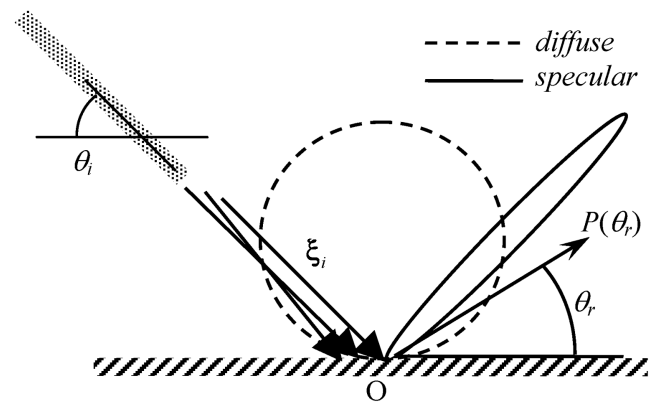

Fig. 1 Schematic of scattering distribution components by Maxwell model.

surface and then evaporates from the surface according to the Maxwellian velocity distribution at the local surface temperature. The Maxwell model considers a fraction $a_{M}$ of the incident molecules to be temporarily absorbed by the surface and then reflected diffusely from the surface; the remaining incident molecules are assumed to reflect specularly.

Scattering distributions (that is, probability distribution plots of gas-surface reflection or scattering angle $\theta_{r}$, the angle between the surface horizon and the molecular velocity upon departure from the surface) illustrate the scattering trends of gas molecules reflected from the solid surface. Polar plots of the scattering distribution predicted by the Maxwell model for a beam of molecules targeted onto a solid surface at a specified angle of incidence are shown in Fig. 1. The part due to specular reflections is a sharp oval and the part due to diffuse reflections is a circle. The composite distribution will then be a circular shape with a protruding peak. If the molecules in the beam are all traveling at the same velocity, then the peak is a line at the specular angle of reflection.

Since Maxwell's model proved suitable for low-speed experiments, such as those by Millikan [8] in 1923, the development of gas-surface interaction models received little attention from the inception of Maxwell's model until satellites began orbiting the Earth and the problem of free-molecular drag brought attention to them [9-13]. Contrary to the expected distributions given by the Maxwell model, high-speed molecular-beam experiments [14-16] showed scattering distributions to be petal-shaped and not centered about the mean specular angle, rather than a composition of a circle and a sharp oval. Partly because of molecular-beam experimental findings, various models were developed to match the observed scattering distributions. For convenience, interaction parameters unique to the model were commonly introduced to examine the model parametrically against laboratory results.

However, free-molecular flow models are inadequate for transitional rarefied conditions. In these conditions, it is necessary to have estimates on reflected velocity distributions and to satisfy the principle of detailed balance for gas-surface interactions, called the reciprocity principle $[17,18]$. The reciprocity principle is fundamental to a formal mathematical approach for relating the reflected distributions to the incident distributions, called scatteringkernel theory [17,19]. In 1971, Cercignani and Lampis [20] initially proposed a model, known as the CL model, that satisfies the fundamental scattering-kernel principles. The CL model involves the tangential momentum and normal kinetic energy accommodation coefficients and performs respectably against laboratory results under certain high-speed rarefied flow conditions [20,21]. Because the CL model has well-defined interaction parameters and compares well in a limited range of laboratory conditions, it remains the preferred scattering-kernel interaction model for engineering analysis and theoretical studies [22], despite efforts to improve the model $[23,24]$.

Twenty years after the CL model was initially published, a transformation of the CL model for use with the DSMC method was laid down by Lord $[7,25,26]$. This transformation is referred to as the CLL model and has now gained wide acceptance; examples of recent applications are reported by Ketsdever and Muntz [27], Santos [28], and Utah and Arai [29]. 
The DSMC method, which reached common acceptance by the 1980 s, relies on gas-surface interaction models at the surface boundaries of the computational domain. For sufficiently low Knudsen and Mach numbers, the diffuse-scattering model suffices. Outside these conditions, such as the initial phase of spacecraft reentry, it is necessary to implement a more sophisticated model for the reasons mentioned already. The two most common models presently in use with the DSMC method are Maxwell's model and the CLL model. It is these two models that are examined in this study. Before describing these models mathematically, two gas-surface interaction modeling concepts are presented.

\section{Mathematical Description of Two Modeling Concepts}

The various gas-surface interaction models that have been proposed since Maxwell's model have resulted in a couple of wellestablished concepts presented here. These concepts are used in describing the two common gas-surface interaction models examined in this study.

\section{A. Interaction Parameters}

Parameters employed in gas-surface interaction models are called interaction parameters. For larger than the nanometer scale, these parameters are relevant to a macroscopic description; typically, they are accommodation coefficients. An accommodation coefficient is a numerical description about the degree to which a flow of gas accommodates kinetically or thermally with a solid surface while interacting with the surface. The accommodation coefficient of molecular property $Q$ is defined in terms of incident and reflected fluxes as follows [30, $\underline{31}]$ :

$$
a_{Q}=\frac{\Phi_{i}^{Q}-\Phi_{r}^{Q}}{\Phi_{i}^{Q}-\Phi_{w}^{Q}}
$$

where $\Phi_{i}^{Q}$ and $\Phi_{r}^{Q}$ are the incident and reflected fluxes of $Q$, respectively, and $\Phi_{w}^{Q}$ is the reflected flux of $Q$, corresponding to full accommodation. Common examples of $Q$ are the total energy $E$, normal momentum $m \xi_{n}$, and tangential momentum $m \xi_{t}$. The total energy, normal momentum, and tangential momentum accommodation coefficients are defined with the usual notation by $\alpha \equiv a_{E}$, $\sigma_{n} \equiv a_{m \xi_{n}}$, and $\sigma_{t} \equiv a_{m \xi_{t}}$, respectively.

\section{B. Scattering Kernel}

In kinetic theory analysis, the gas-surface interaction model is used as a boundary condition. A formal mathematical construct for a gas-surface interaction model is the scattering-kernel formulation outlined, for example, by Cercignani [32]. A scattering kernel $K\left(\boldsymbol{\xi}_{i}, \boldsymbol{\xi}_{r}\right)$ represents the probability density that an incident molecule with velocity $\boldsymbol{\xi}_{i}$ is reflected with velocity $\boldsymbol{\xi}_{r}$ at essentially the same time and place. It bridges the velocity distribution functions $f_{i}\left(\boldsymbol{\xi}_{i}\right)$ and $f_{r}\left(\xi_{r}\right)$ of the incident and reflected molecules, respectively, through the following integral transform:

$$
\xi_{n, r} f_{r}\left(\boldsymbol{\xi}_{r}\right)=\int_{\xi_{n, i}<0}\left|\xi_{n, i}\right| f_{i}\left(\boldsymbol{\xi}_{i}\right) K\left(\boldsymbol{\xi}_{i}, \boldsymbol{\xi}_{r}\right) \mathrm{d} \boldsymbol{\xi}_{i}
$$

where each gas-surface interaction is independent of others, and the average interaction time is small relative to the temporal evolution of $f$. In addition, the scattering kernel satisfies the following three criteria: positivity, normalization, and reciprocity. These criteria are expressed mathematically as follows:

$$
\begin{gathered}
K\left(\boldsymbol{\xi}_{i}, \boldsymbol{\xi}_{r}\right) \geq 0 \\
\int_{\xi_{n, r}>0} K\left(\boldsymbol{\xi}_{i}, \boldsymbol{\xi}_{r}\right) \mathrm{d} \boldsymbol{\xi}_{r}=1 \\
\left|\xi_{n, i}\right| f_{M}\left(\boldsymbol{\xi}_{i}\right) K\left(\boldsymbol{\xi}_{i}, \boldsymbol{\xi}_{r}\right)=\left|\xi_{n, r}\right| f_{M}\left(\boldsymbol{\xi}_{r}\right) K\left(-\boldsymbol{\xi}_{i},-\boldsymbol{\xi}_{r}\right)
\end{gathered}
$$

where $f_{M}$ is the Maxwellian velocity distribution in equilibrium with the solid surface:

$$
f_{M}(\xi) \mathrm{d} \xi=\left(2 \pi R_{G} T_{w}\right)^{-\frac{3}{2}} \exp \left(-\frac{\xi^{2}}{2 R_{G} T_{w}}\right) \mathrm{d} \xi
$$

The product $f(\xi) \mathrm{d} \xi$ is the probability of any given molecule to have velocity $\xi$ within the range from $\xi$ to $\xi+\mathrm{d} \xi$. The reciprocity condition $[17,18]$ [Eq. (ㄷ)] is the equilibrium condition for gassurface interactions. It must be satisfied when the flow is in equilibrium with the solid surface.

\section{Mathematical Description of Two Common Models in Use with DSMC}

\section{A. Maxwell Model}

The most common model in use with the DSMC method is the Maxwell model. It has a scattering kernel that satisfies positivity, normalization, and reciprocity and is written as follows [19]:

$$
K_{M}\left(\boldsymbol{\xi}_{i}, \boldsymbol{\xi}_{r}\right)=\left(1-a_{M}\right) \delta\left(\boldsymbol{\xi}_{i}-\boldsymbol{\xi}_{r, \text { specular }}\right)+a_{M} f_{M}\left(\boldsymbol{\xi}_{r}\right)\left|\boldsymbol{\xi}_{r} \cdot \mathbf{n}\right|
$$

where $\boldsymbol{\xi}_{r, \text { specular }}$ is the molecular velocity of specular reflection, and $a_{M}$ is Maxwell's fraction, as described in Sec. II. It is an accommodation coefficient that indicates the probability of a diffuse reflection and does not represent a ratio of fluxes. In the implementation within the MONACO DSMC code, a reflected molecule's internal energy for a diffuse reflection is computed based on thermal equilibrium with the local surface temperature. For a specular reflection, the molecule's internal energy is assumed to be unchanged. In other words, in MONACO, $a_{E_{\text {int }}}=a_{M}$ for the Maxwell model.

\section{B. Cercignani, Lampis, and Lord Model}

As discussed in Sec. II, the best probabilistic gas-surface interaction model is the $C \bar{L}$ model because it has been shown to match certain laboratory scattering distributions, it involves welldefined interaction parameters [namely, parameters that can be expressed in the form of Eq. (1)], and it involves a well-defined mathematical framework (namely, the scattering-kernel construction). The CL model interaction parameters are the accommodation coefficient for the tangential momentum $\sigma_{t} \equiv a_{Q}$, where $Q=m \boldsymbol{\xi} \cdot \mathbf{t}$, and the accommodation coefficient for the normal part of the kinetic energy $\alpha_{n} \equiv a_{Q}$, where $Q=\frac{1}{2} m(\xi \cdot \mathbf{n})^{2}$. The scattering kernel has the following form:

$$
\begin{aligned}
& K_{\mathrm{CL}}\left(\boldsymbol{\xi}_{i}, \boldsymbol{\xi}_{r}\right)=\frac{\left[\alpha_{n} \sigma_{t}\left(2-\sigma_{t}\right)\right]^{-1}}{2 \pi\left(R_{G} T_{w}^{2}\right)} \xi_{n, r} \exp \left(-\frac{\xi_{n, r}^{2}+\left(1-\alpha_{n}\right) \xi_{n, i}^{2}}{2 \alpha_{n} R_{G} T_{w}}\right) \\
& \quad \times \exp \left(-\frac{\left[\boldsymbol{\xi}_{t, r}-\left(1-\sigma_{t}\right) \boldsymbol{\xi}_{t, i}\right]^{2}}{2 \sigma_{t}\left(2-\sigma_{t}\right) R_{G} T_{w}}\right) I_{0}\left(\frac{\sqrt{1-\alpha_{n}} \xi_{n, r} \xi_{n, i}}{\alpha_{n} R_{G} T_{w}}\right)
\end{aligned}
$$

where $\xi_{n, i}<0, \xi_{n, r}>0,0 \leq \sigma_{t} \leq 2,0 \leq \alpha_{n} \leq 1, I_{0}$ is the modified Bessel function of the first kind and of zeroth order, and $\xi_{t}$ is the vector sum of the tangential components of velocity.

The CL kernel, the scattering kernel of the CL model, is implemented into a DSMC code by a simple algorithm with a level of complexity not much greater than the implementation of the Maxwell model. Lord [7] originally made the transformation to this algorithm with the help of a graphical representation of the CL model. This algorithm forms the basic CLL model and is commonly employed with DSMC. Table 1 presents the algorithm equations that are employed by the MONAC $\mathrm{CO}$ DSMC code. In these equations, $\alpha_{t} \equiv a_{Q}$, where $Q=\frac{1}{2} m\left(\xi \cdot \mathbf{t}_{i}\right)^{2}$, and $\mathbf{t}_{i}$ is any surface tangent vector. In other words, $\alpha_{t}$ is the accommodation coefficient for the part of the kinetic energy along any tangent to the surface; it is assumed to be independent of direction along the surface. 
Table 1 Algorithm equations - of the CLL model for computing $\xi_{r}$

\begin{tabular}{ccc}
\hline \hline Normal component & Tangent 1 component & Tangent 2 component \\
\hline$r_{1}=\sqrt{-\alpha_{n} \ln x_{1}}$ & $r_{3}=\sqrt{-\alpha_{t} \ln x_{3}}$ & - \\
$\phi_{2}=2 \pi x_{2}$ & $\phi_{4}=2 \pi x_{4}$ & $r_{5}=\sqrt{-\alpha_{t} \ln x_{5}}$ \\
$\xi_{n, m}=\left|\xi_{n, i} / \xi_{\mathrm{mp}, w}^{\prime}\right| \sqrt{1-\alpha_{n}}$ & $\xi_{t, m}=\left|\xi_{t 1, i} / \xi_{\mathrm{mp}, w}^{\prime}\right| \sqrt{1-\alpha_{t}}$ & $\phi_{6}=2 \pi x_{6}$ \\
$\xi_{n, r}=\xi_{\mathrm{mp}, w}^{\prime} \sqrt{r_{1}^{2}+\xi_{n, m}^{2}+2 r_{1} \xi_{n, m} \cos \phi_{2}}$ & $\xi_{t 1, r}=\xi_{\mathrm{mp}, w}^{\prime}\left(\xi_{t, m}+r_{3} \cos \phi_{4}\right)$ & $\xi_{t 2, r}=\xi_{\mathrm{mp}, w}^{\prime} r_{5} \cos \phi_{6}$ \\
\hline \hline
\end{tabular}

${ }^{\mathrm{a} A u x i l i a r y}$ information: $x_{i}$ are random numbers uniformly distributed between 0 and $1, \xi_{\mathrm{mp}, w}^{\prime}=\sqrt{2 R_{G} T_{w}}$, and $\alpha_{t}=\sigma_{t}\left(2-\sigma_{t}\right)$.

\section{Flat-Plate Wind-Tunnel-Test Simulations Using the Two Models}

\section{A. General Description}

To examine the gas-surface interaction models, computer simulations are performed of existing wind-tunnel tests examining rarefied hypersonic flow over a flat plate. The wind-tunnel tests reported by Cecil and McDaniel [33] in 2005 use planar-laserinduced fluorescence (PLIF) of seeded iodine within a free-jet expansion of nitrogen to measure boundary-layer velocity over a flat plate. The plate is formed by the flat side of a wedge made of opticalquality fused quartz. The measured velocity represents the mean value of the molecular velocity distribution. Further details of the wind-tunnel test-section apparatus and flat-plate model are presented by Cecil and McDaniel.

The computer simulations of the flowfield are performed assuming two-dimensional flow. In addition, the simulations use rectangular spatial domains that cover a region near the leading edge of the flat plate, which includes an adequate number of wind-tunneltest measurement locations. Each domain is divided into quadrilateral cells to form two-dimensional computational meshes. Figure 2 illustrates the computational mesh and its location relative to the flat-plate model. From this perspective, the simulation particles traverse the mesh from left to right. The inflow boundary is the left vertical edge of the mesh, $2 \mathrm{~mm}$ upstream of the leading edge. Computational cells are adapted so that they are about the size of the local mean free path. A finer mesh indicates that the grid resolution is sufficient. Throughout each mesh, a uniform particle weight $W_{p}$ is assigned. The meshes are generated with HyperMesh [34].

To simulate the experiment, pure nitrogen is assumed. The corresponding input parameters for the nitrogen gas are a molecular weight of 28.01, number of rotational energy degrees of freedom $\zeta_{\text {rot }}=2.0$, number of vibrational energy degrees of freedom $\zeta_{\text {vib }}=0.0$, characteristic temperature of vibration $\theta_{\text {vib }}=3390 \mathrm{~K}$, characteristic temperature of the intermolecular potential $T^{*}=91.5 \mathrm{~K}$, and maximum rotational collision number $Z_{\mathrm{rot}, \infty}=18.1$. For collision cross-sectional data, the parameters are the characteristic molecular diameter $\delta_{\text {diameter }}=4.11 \times 10^{-10} \mathrm{~m}$ and the viscosity index of the variable-hard-sphere collision model $\omega_{11}=$ 0.7 for collisions between nitrogen molecules. For solid-surface data, the parameter is the temperature of the solid-surface wall $T_{w}=300 \mathrm{~K}$.

The velocity profiles at the simulation inflow boundary are shown in Fig. 3. These profiles are provided by Cecil and McDaniel [33]. The translational temperature $T_{\text {tra }}$ and number density $n$ inflow profiles are calculated from the inflow velocity profiles and the windtunnel-test reservoir temperature and pressure, $T_{0}=300 \mathrm{~K}$ and $p_{0}=1.79 \mathrm{~atm}$, respectively. The calculation of $T_{\text {tra }}$ and $n$ assumes

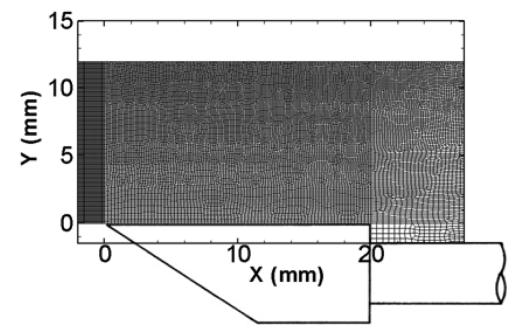

Fig. 2 Two-dimensional simulation mesh and its location relative to the flat-plate model. that the reservoir contains an ideal gas and that it is expelled isentropically as a free-jet expansion. The global Knudsen number is about 0.004, which is in the near-continuum regime. This is based on the inflow centerline (ICL) mean free path $\left(\lambda_{\text {ICL }} \approx 0.08 \mathrm{~mm}\right)$ and the flat-plate length $(l=20 \mathrm{~mm})$.

Using these flow conditions, simulations are performed with the Maxwell gas-surface interaction model at various values of Maxwell's fraction: $a_{M}=0,0.25,0.50,0.75$, and 1.00. Simulations are also conducted with the CLL gas-surface interaction model at various values of tangential momentum accommodation $\left(\sigma_{t}=0\right.$, $0.25,0.50,0.75$, and 1.00 ) with full normal kinetic energy accommodation $\left(\alpha_{n}=1.00\right)$. In addition, the sensitivity to normal kinetic energy accommodation is examined with similar levels of accommodation $\left(\alpha_{n}=0,0.25,0.50,0.75\right.$, and 1.00) while maintaining zero tangential momentum accommodation $\left(\sigma_{t}=0\right)$. Each of the simulations with the CLL model assumes full internal energy accommodation $\left(a_{E_{\text {int }}}=1\right)$. All of these simulations require computational parameters similar to the following: $W_{p}=$ $1.0 \times 10^{12}, \sim 1,915,000$ particles, 19,067 cells, 4 processors (1.4 GHz AMD Opteron 240 or $2.8 \mathrm{GHz}$ AMD Opteron 254, $1 \mathrm{~GB}$ RAM per processor), time-step size of $1.0 \times 10^{-8} \mathrm{~s}, 75,000$ time steps, and wall time of $\sim 3.5 \mathrm{~h}$.

The local Knudsen number along the flat-plate surface provides a further indication of the level of flow rarefaction. Figure 4 is a plot of the gradient-length local Knudsen number based on the local density

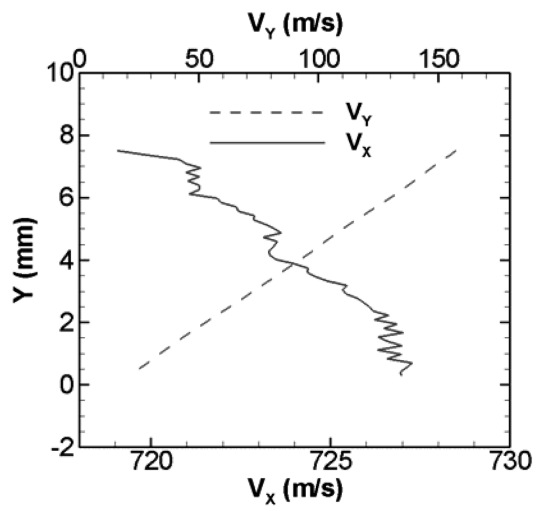

Fig. 3 Nonuniform inflow velocity for DSMC simulations of flat-plate wind-tunnel test.

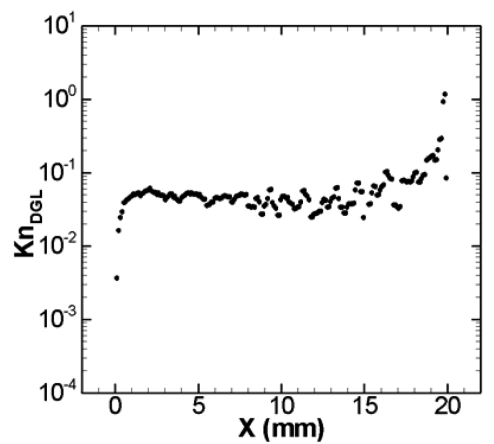

Fig. 4 Density-gradient-length local Knudsen number along the flatplate surface with full accommodation. 
gradient next to the flat plate:

$$
K n_{\text {DGL }}=\frac{\lambda}{\rho} \frac{\mathrm{d} \rho}{\mathrm{d} x}
$$

These data are extracted from a simulation using the Maxwell gassurface interaction model with $a_{M}=1.0$. At the leading edge $(x=0 \mathrm{~mm})$, the local Knudsen number has a near-continuum value of about $3 \times 10^{-3}$. Moving along the surface, it quickly increases to a near rarefied value of about $5 \times 10^{-2}$. Approaching the end of the plate, the value increases into the rarefied regime. The flow expands at the leading edge because of an oblique shock wave and at the trailing edge because of the sudden end of the flat plate. The range of Knudsen number indicates that the DSMC method is appropriate for simulating the flowfield.

\section{B. Effects of Gas-Surface Modeling on the Velocity Field}

The variation of Maxwell's fraction $a_{M}$ from fully specular to fully diffuse results in a variation in boundary-layer size from nonexistent to maximum extent. Part of this variation is illustrated by the halfand fully diffuse cases in Figs. $5 \mathrm{a}$ and $5 \mathrm{~b}$. In these figures, streamlines of bulk flow velocity diverge from the left boundary because the inflow velocity field is expanding from an axisymmetric nozzle. They also diverge near the surface because of the growing shock layer underneath the oblique shock wave. The origin of the coordinate system is set at the leading edge of the flat plate. A similar variation in boundary-layer size is given by the parametric analysis with the CLL model involving the variation in tangential momentum accommodation $\sigma_{t}$, with full normal kinetic energy accommodation $\alpha_{n}=1$, except that the boundary layer does not completely disappear at $\sigma_{t}=0$.

\section{Effects of Gas-Surface Modeling on Molecular Probability Distributions}

Molecular distribution plots provide a detailed perspective of the effects of the different gas-surface interaction models and of varying the gas-surface accommodation. Probability distributions of molecular velocity at two locations near the leading edge of the flat-plate surface are examined. The molecular velocity distributions at $x=0 \mathrm{~mm}$ are unaffected by changes in gas-surface interaction model or gas-surface accommodation level. These distributions correspond to the first computational cell over the flat-plate surface, which begins at $x=0 \mathrm{~mm}$ and ends at $x=0.25 \mathrm{~mm}$; this cell and all other cells next to the surface are about $0.1 \mathrm{~mm}$ high. They are unaffected by changes in accommodation because they are associated with a computational cell that is within the first mean free path over the flat plate. Molecular velocity distributions of the computational cell containing point $(x, y)=(5.0,0.0) \mathrm{mm}$ are shown in Figs. $\underline{6 a}$ and $\underline{6 \mathrm{~b}}\left(a_{M}=\sigma_{t}=0,0.25,0.5,0.75,1.0\right)$. This figure illustrates that the degree of translational nonequilibrium next to the surface is proportional to the level of gas-surface accommodation. It also shows that the Maxwell and CLL models give identical results only for full gas-surface accommodation, $a_{M}=\sigma_{t}=1.0$. At partial levels of gas-surface accommodation, the models yield similar $\xi_{x}$ distributions. However, the $\xi_{y}$ distributions at $\xi_{y}>0$ are significantly different. This is because the accommodation coefficient regulating normal kinetic energy $\alpha_{n}$ is held constant at unity in the CLL model, whereas in the Maxwell model, the normal kinetic energy accommodation is varied implicitly through $a_{M}$.

The molecular surface scattering plots are also examined at the two positions $(x=0$ and $5.0 \mathrm{~mm})$ over the flat-plate surface. These are probability distribution plots of reflected molecule scattering angle $\theta_{r}$. Figures $7 \mathrm{a}$ and $7 \mathrm{~b}$ present the distributions. The scattering plots show the essential differences between the Maxwell and CLL gassurface interaction models. The Maxwell scattering distributions
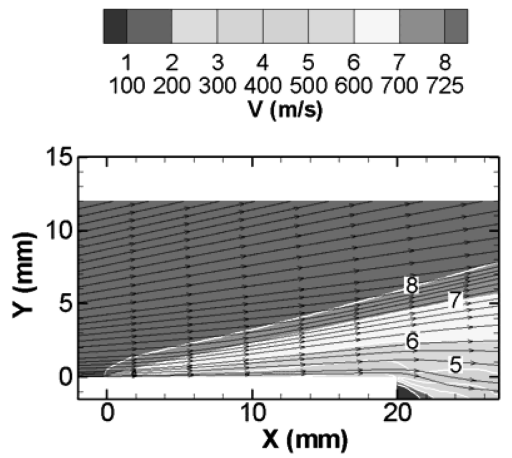

a) $a_{M}=0.5$
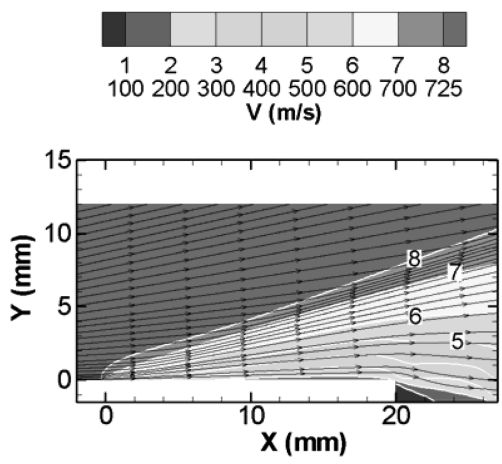

b) $a_{M}=\mathbf{1 . 0}$

Fig. 5 Flow-speed contours and streamlines over the flat plate by DSMC.

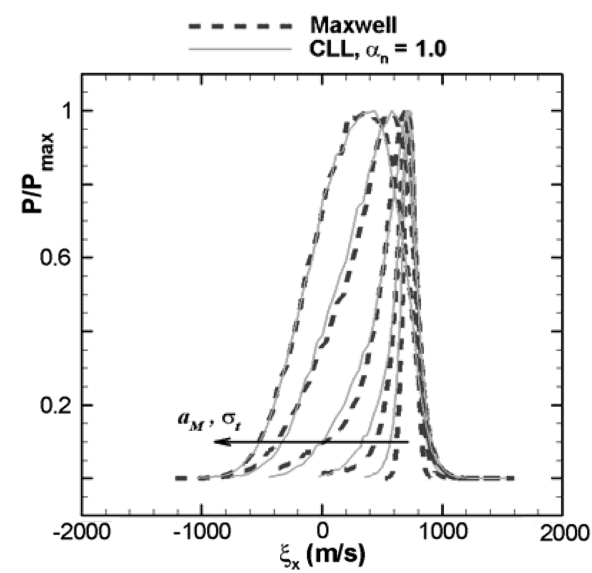

a) Parallel component distributions

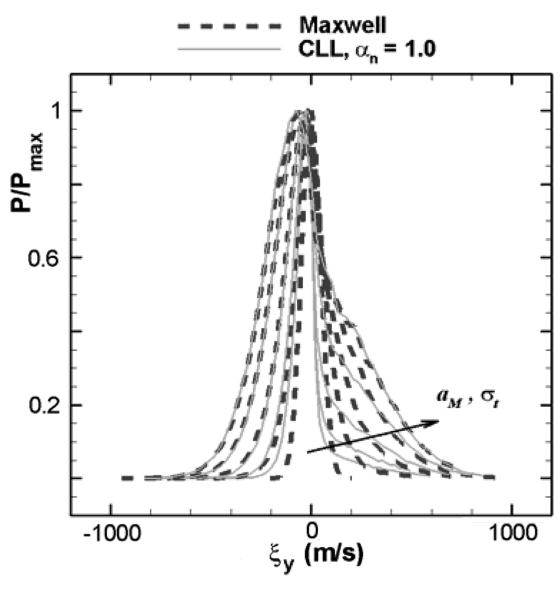

b) Perpendicular component distributions

Fig. 6 Molecular velocity distributions of computational cell next to the flat plate at $x=5.0 \mathrm{~mm}$. 


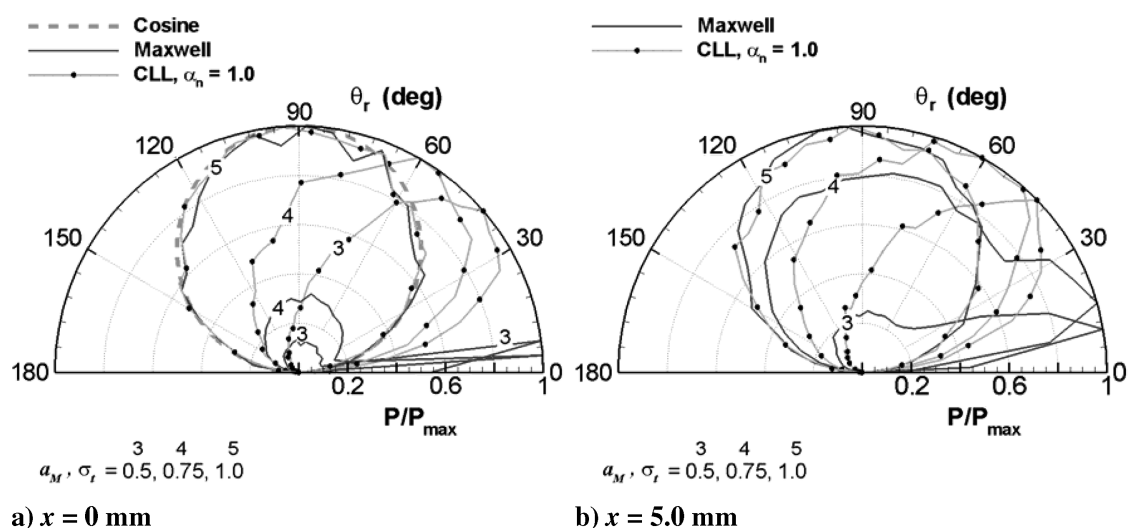

Fig. 7 Scattering distributions at two locations on the flat-plate surface.

have unrealistic peaks due to the specular angle at partial levels of accommodation; the associated diffuse component of the scattering distribution is evident by the circular segments. The CLL scattering distributions are petal-shaped, similar to observations of reflected rarefied molecular beams from clean flat surfaces [14,15]. As required, the scatter plots further confirm that both models are equivalent at full accommodation. There, they yield the Lambert or cosine distribution of optics theory, which also applies to the random distribution of gas-surface scattering angles. These plots also show variations in scattering due to variations in accommodation and position $x$ along the flat-plate surface. The Maxwell distributions have abrupt changes with increasing accommodation and position; in contrast, the CLL distributions vary smoothly with accommodation and position.

\section{Determination of Flat-Plate Gas-Surface Accommodation}

For each of the simulations, data are extracted along vertical slices corresponding to the locations at which PLIF measurements were taken: $x=0,2.5,5,7.5,10,12.5,15,17.5$, and $20 \mathrm{~mm}$ from the leading edge. These data include $x$ and $y$ velocity components. Figures $\underline{8 \mathrm{a}}-\underline{\mathrm{d}}$ present some of the comparisons between the DSMC parametric results and the PLIF data. At the leading edge of the flat plate, the DSMC simulations match the experimental data; hence, the
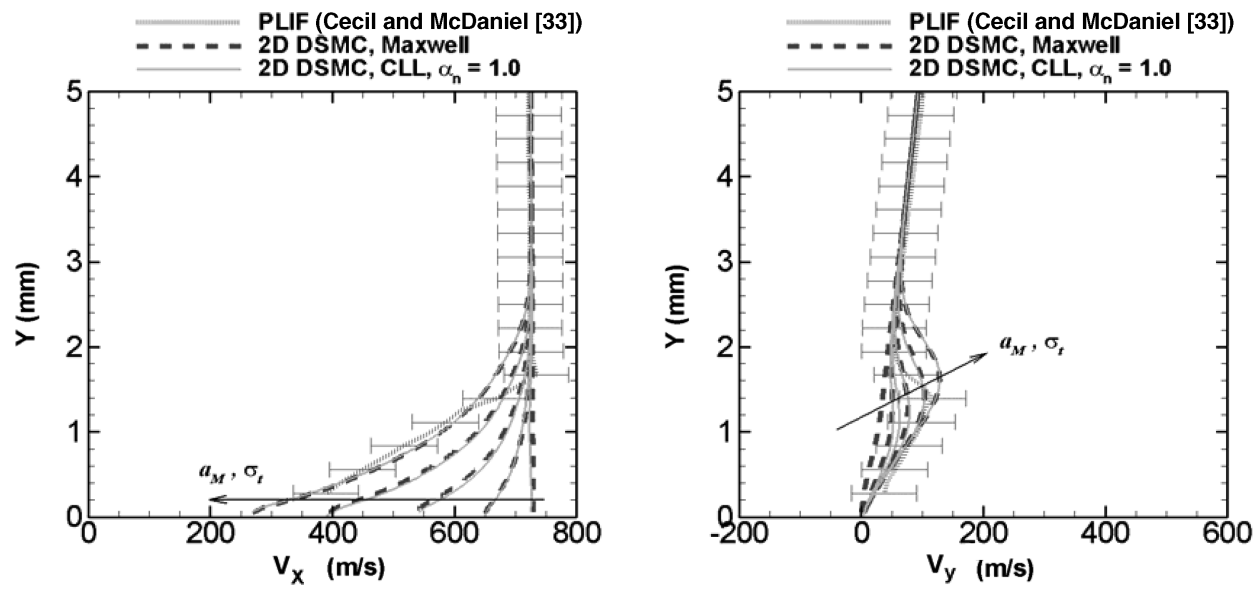

a) $V_{x}$ profiles, $x=5.0 \mathrm{~mm}$

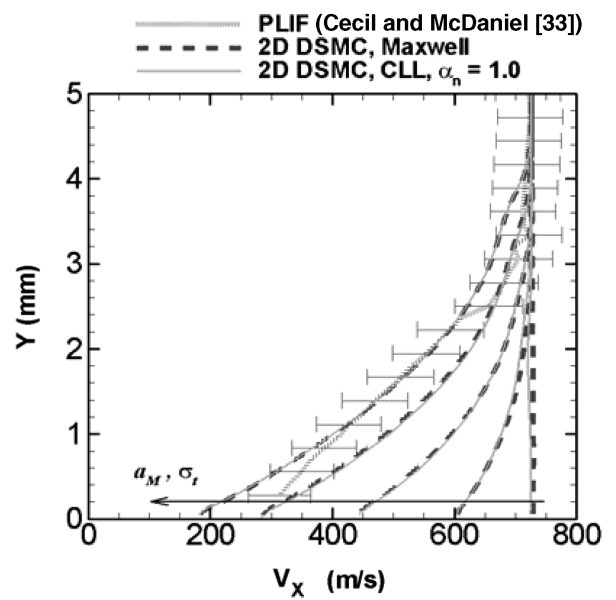

c) $V_{x}$ profiles, $x=10 \mathrm{~mm}$

b) $V_{y}$ profiles, $x=5.0 \mathrm{~mm}$

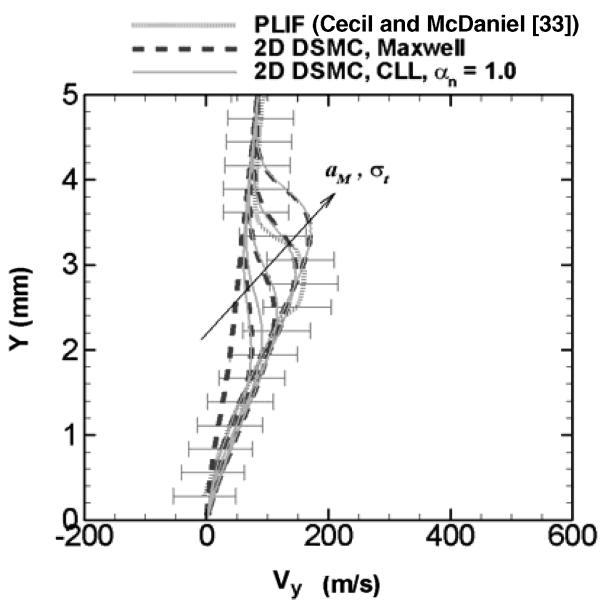

d) $V_{y}$ profiles, $x=10 \mathrm{~mm}$

Fig. 8 Comparison of flat-plate boundary-layer velocity profiles, $a_{M}$ and $\sigma_{t}=0,0.25,0.5,0.75$, and 1.0. 
correct inflow conditions are employed. Furthermore, the DSMC and wind-tunnel data agree in the freestream region above the boundary layer.

The boundary layer is where the flowfield is affected by different gas-surface interaction models and different levels of gas-surface accommodation. When full gas-surface accommodation conditions are specified, by setting all accommodation coefficients equal to one, the Maxwell and CLL models provide the same boundary-layer profiles as expected from the definition of the models. In addition, the Maxwell and CLL models yield essentially the same results for $a_{M}=\sigma_{t}=0.5$ and 0.75. However, Maxwell and CLL models differ significantly at $a_{M}=\sigma_{t}=0$ and 0.25 . Hence, the Maxwell and CLL models yield the same velocity boundary-layer profiles when $a_{M}=$ $\sigma_{t} \geq 0.5$ for these flow conditions and $a_{E_{\text {int }}}=\alpha_{n}=1.00$ in the CLL model.

The simulations involving full gas-surface accommodation $\left(a_{M}=\sigma_{t}=1.0\right)$ give the overall best agreement with the measured data for the $V_{x}$ profiles, except at the transition from the boundary layer to the freestream, where the diffuse shock lies. The DSMC velocity profiles, even for the full-accommodation case, disagree with the measured data at $x \geq 15 \mathrm{~mm}$. This is due to an adverse pressure gradient that is believed to be caused by wind-tunnel-test flow phenomena, beyond the specified computational domain, and thus not captured by the simulation. To estimate the gas-surface accommodation, the profiles at $x \leq 12.5 \mathrm{~mm}$ are examined, which are unaffected by the adverse pressure gradient. The simulations with $a_{M}=\sigma_{t}=0.75$ provide the overall best agreement with the measured data for the $V_{y}$ profiles and the $V_{x}$ profiles in the transition from the boundary layer to the freestream. Hence, the level of accommodation that provides the overall best agreement among both the $V_{x}$ and $V_{y}$ profiles is a compromise between 0.75 and 1.0. Simulations with the average accommodation $\left(a_{M}=\sigma_{t}=0.875\right)$ result in profiles lying in the middle, in accord with the linear

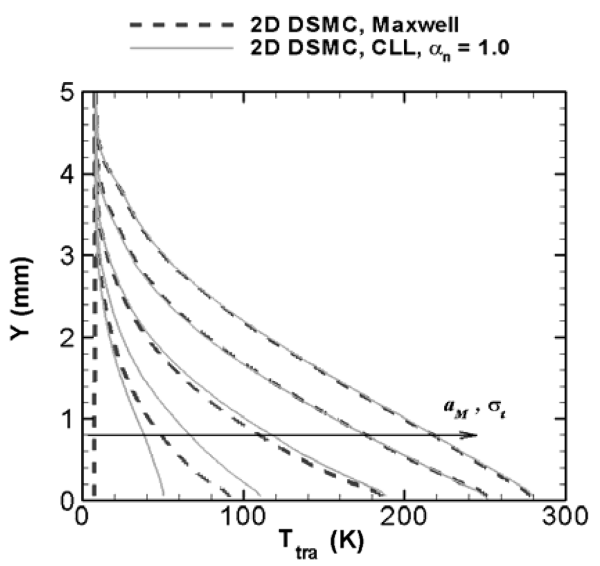

a) Translational temperature

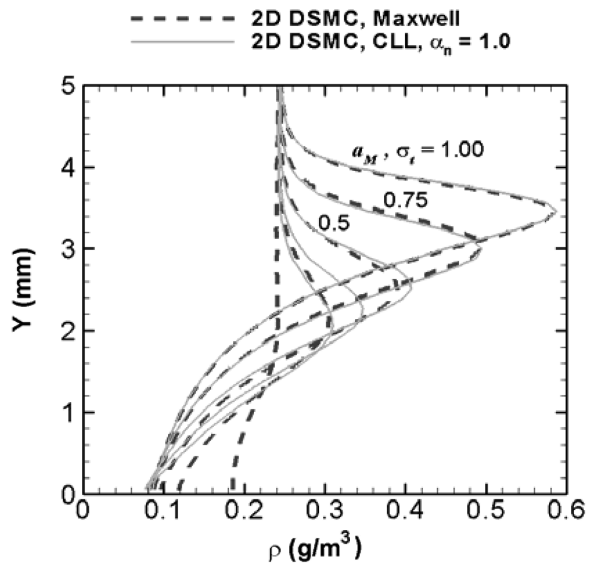

c) Density variation of the profiles with accommodation, when the accommodation level is greater than 0.5 . Hence, the overall best agreement among the considered accommodation levels of the $a_{M}$ and $\sigma_{t}$ sensitivity studies occurs when $a_{M}=\sigma_{t} \approx 0.90$, the value being rounded according to the uncertainty in the result of the optimization.

The prediction of $90 \%$ accommodation is reasonable because of the relatively high gas density, similar to density of air at $60 \mathrm{~km}$ altitude, according to U.S. 1976 standard atmosphere [35]. Recall that the accommodation coefficient is a macroscopic property defined in terms of local incident and reflected fluxes. When the density is high, it is more probable that reflected gas molecules reencounter the solid surface within a microscopic time scale, because of collisions with other gas molecules near the surface. The prediction of accommodation is also reasonable from laboratory measurements. For example, Cook et al. [36] measured 60 to $80 \%$ tangential momentum accommodation in experiments involving rarefied molecular beams of nitrogen with a mean incident velocity of $1300 \mathrm{~m} / \mathrm{s}$ that are scattered by a silicon cover plate for a satellite solar cell. Accommodation coefficient increases with a decrease in incident mean velocity. The present case involves higher accommodation associated with lower incident velocities of nitrogen against optical-quality fused quartz.

The sensitivity study of normal kinetic energy accommodation coefficient $\alpha_{n}$ confirms that $V_{x}$, the tangential component of velocity, is not significantly affected. The variation of $\alpha_{n}$ does affect $V_{y}$, the normal component of velocity, but to an order-of-magnitude-smaller extent than the same variation of $\sigma_{t}$. This study indicates that it is possible to improve the agreement in $V_{y}$ between the CLL simulation with $\sigma_{t}=1.0$ and the measured data by significantly reducing $\alpha_{n}$; however, there is no indication in the experiments found in the literature, or even in physical reasoning, that low normal kinetic energy accommodation would occur along with full tangential

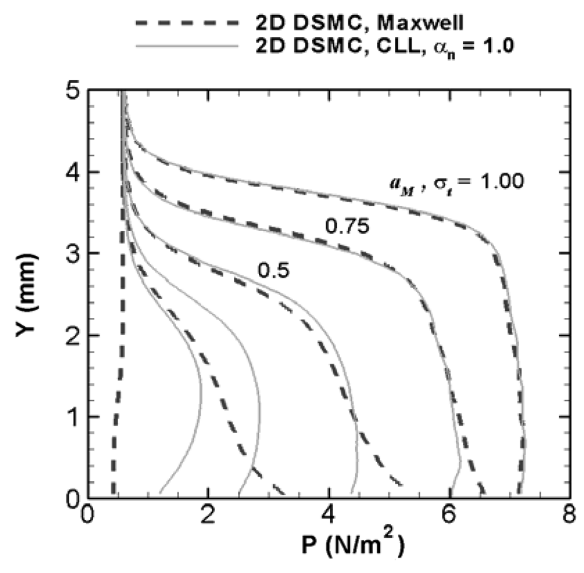

b) Pressure

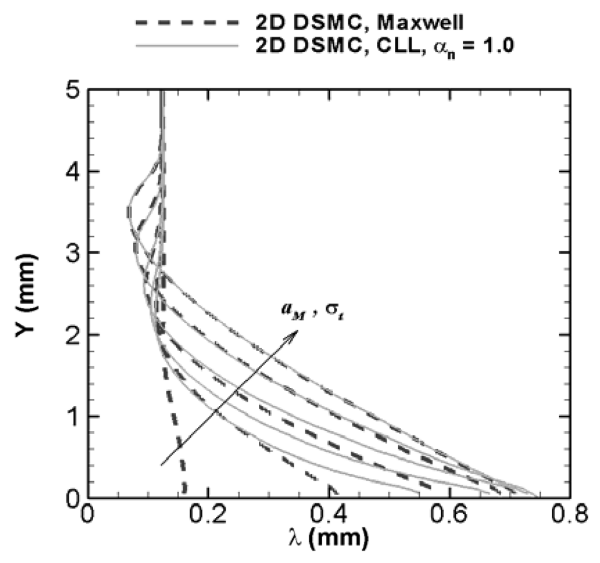

d) Mean free path

Fig. 9 Comparison of flat-plate boundary-layer profiles of several flow properties, $a_{M}$ and $\sigma_{t}=0,0.25,0.5,0.75$, and 1.0, $x=10.0 \mathrm{~mm}$. 
momentum accommodation $\sigma_{t}$. Gombosi [31] stated that the normal accommodation is relatively unknown and usually assumed to be equal to unity. Hence, this case can not be favored over the $\sigma_{t}=0.90$ case.

\section{E. Effects of Gas-Surface Modeling on Boundary-Layer and Surface Aerothermodynamics}

In addition to velocity, boundary-layer profiles of translational temperature, pressure, density, and mean free path are examined. Figures $9 \mathrm{a}-9 \mathrm{~d}$ display these profiles at $x=10 \mathrm{~mm}$ from the leading edge. The $\overline{M a x w e l l}$ and CLL results are again similar at $a_{M}$ and $\sigma_{t}=1.00$ and 0.75 ; however, differences are clearly noticeable at $a_{M}$ and $\sigma_{t}=0.5$. In this analysis, the translational temperatures are different at the lower accommodation levels, because the accommodation coefficient of the normal component of kinetic energy $\alpha_{n}$ and the internal energy accommodation coefficient $a_{E_{\text {int }}}$ are fixed at unity for the CLL model, whereas they are implicitly varied as part of Maxwell's fraction $a_{M}$ in the Maxwell model. Near the surface, the pressure profiles differ at partial accommodation levels, because the models have distinct scattering distributions. As the gas-surface accommodation is lowered, this effect is greater, because the scattering distributions are more distinct. The factors affecting the temperature and pressure profiles also affect the density and mean free path profiles because the properties are linked through thermodynamic and kinetic principles.

The shape of the profiles describe boundary-layer flow behavior. The pressure and density profiles are sensitive to the presence of a diffuse shock. At full accommodation, the pressure profile provides a clear illustration. It suddenly rises across the shock and then remains essentially constant within the boundary layer. The mean free path in the shock is, at most, roughly $50 \%$ smaller than in the freestream. Descending below the shock, $\lambda$ continually grows until reaching the

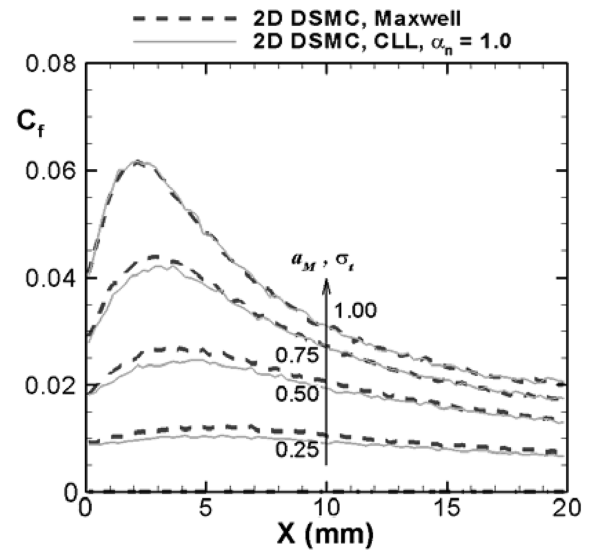

a) Skin friction coefficient

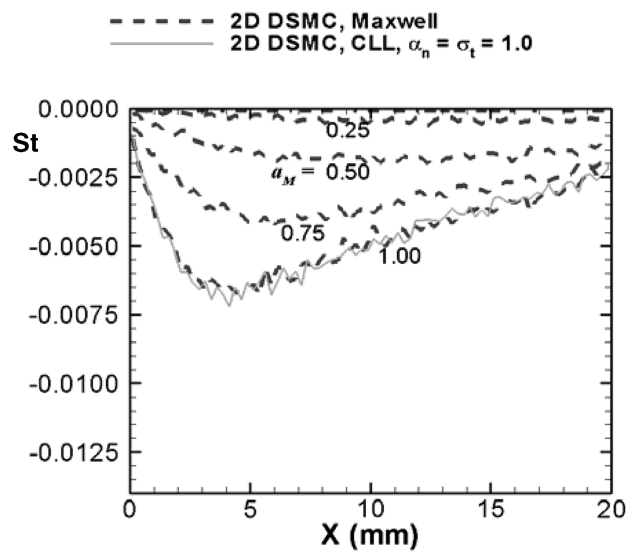

c) Stanton number, Maxwell model surface because of flow expansion below the diffuse oblique shock. At full accommodation, $\lambda$ next to the surface is 7.5 times larger than within the shock. The temperature profiles show a smooth change from freestream to surface conditions, with no significant indication of the presence of a diffuse shock. Rarefied flow behavior is also revealed by the temperature jump at the surface, which is no smaller than $20 \mathrm{~K}$.

Finally, the affects of gas-surface modeling on surface properties are examined. These are the distributions of pressure coefficient, skin-friction coefficient, and Stanton number along the surface. Figures 10a-10d present these distributions. For the skin-friction coefficient, the Maxwell and CLL results have small differences. The differences occur because the CLL model has accommodation coefficients associated with normal and tangential momentum flux. Skin friction is related to the difference between the incident and reflected tangential momentum flux. The majority of the momentum in the flow is directed tangentially to the surface. Consequently, varying tangential momentum accommodation nearly affects the skin-friction profiles the same as varying total momentum accommodation. For this reason, the parametric study of the Maxwell and CLL models produces similar skin-friction profiles. For the pressure coefficient, the agreement occurs at $a_{M}$ and $\sigma_{t} \geq 0.5$. The differences below $50 \%$ accommodation are a result of maintaining $\alpha_{n}=1$ in the CLL model, whereas it is implicitly varied as part of Maxwell's fraction $a_{M}$ in the Maxwell model.

For the Stanton number, the models agree only at full accommodation. The Stanton number is nondimensionalized heat flux. Negative values indicate that heat is transferred from the surface to the gas. The heat flux is determined by the difference between the incident and reflected kinetic and internal energy fluxes. When $a_{M}=0$ in Maxwell's model, the incident and reflected energy fluxes are equal; hence, the Stanton number is zero. When $\sigma_{t}=0$ in the CLL

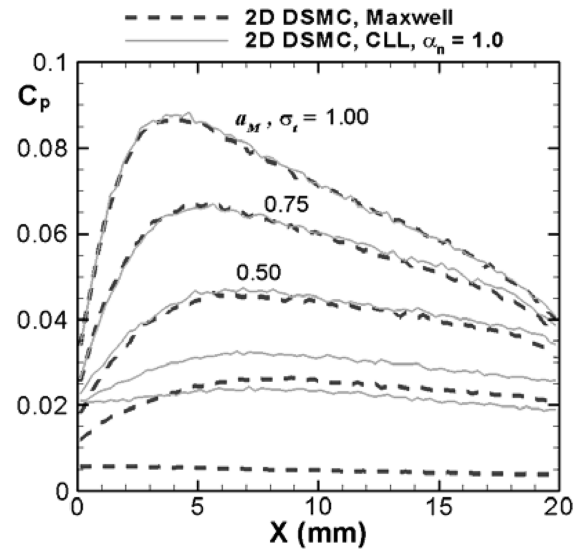

b) Pressure coefficient

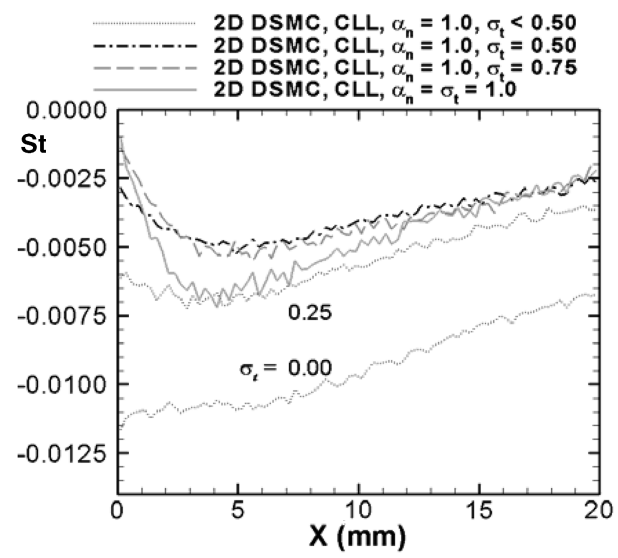

d) Stanton number, CLL model

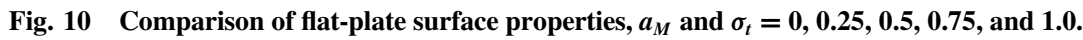


model, the tangential component of velocity is reflected specularly and the difference between the incident and reflected fluxes of tangential component of kinetic energy is zero. However, full internal energy accommodation $a_{E_{\text {int }}}$ and normal kinetic energy accommodation $\alpha_{n}$ is assumed. Thus, as $\sigma_{t}$ is lowered, the difference between the incident and reflected fluxes of the normal component of kinetic energy and of internal energy is increasingly revealed. Hence, these simulations result in different trends in Stanton number distributions for the CLL and Maxwell models.

\section{Conclusions}

This study began with a review of gas-surface interaction models since the Maxwell model. The review led to the decision to assess the Maxwell and the CLL gas-surface interaction models, because they are the two most common models in use with DSMC. Then mathematical descriptions of these models were given. This was followed by a description of the DSMC simulations of an existing flat-plate wind-tunnel-test study [33]. The gas-surface interaction models were assessed using these $\overline{\mathrm{DS}} \mathrm{MC}$ simulations by examining molecular distributions, boundary-layer profiles, and surface properties.

Molecular probability distributions were analyzed near the leading edge. For molecular velocity distributions, the Maxwell and CLL gas-surface interaction models gave distinct $\xi_{y}$ distributions at partial levels of gas-surface accommodation. For scattering distributions, the Maxwell model had unrealistic peaks due to specular reflection, whereas the CLL model had petal-shaped distributions, similar to observations of molecular-beam studies reported in the literature. It was also determined that the Maxwell scattering distributions experienced abrupt changes with increasing accommodation and position, whereas the CLL distributions varied smoothly.

Boundary-layer profiles were examined at selected locations from the leading edge. For boundary-layer velocity profiles, the Maxwell and CLL models gave mostly similar boundary-layer profiles from 50 to $100 \%$ gas-surface accommodation. Approximately $90 \%$ gassurface accommodation yielded the overall best agreement between the simulations and the measured velocity data, which is consistent with physical reasoning and the general trends found in the literature. Temperature, pressure, and density boundary-layer profiles and surface-property distributions of pressure, skin friction, and heat flux further illustrated the effects of the different scattering distributions and accommodation coefficients of the Maxwell and CLL models.

The Maxwell and CLL gas-surface interaction models provide similar aerodynamic results at accommodation levels greater than $50 \%$. Thus, they produce similar aerodynamic results for the present flat-plate conditions. Normal kinetic energy and internal energy accommodation play a significant role in heat transfer. Further study is necessary to determine the adequacy of the models for heat transfer prediction.

\section{Acknowledgments}

This work is sponsored by the Space Vehicle Technology Institute under NASA grant NCC-3989 with joint sponsorship from the U.S. Department of Defense. The authors gratefully acknowledge windtunnel data provided by Eric Cecil and James McDaniel from the University of Virginia.

\section{References}

[1] FAI Sporting Code, Section 8 [online database], http://www.fai.org/ sporting_code/sc8.asp [retrieved June 2008].

[2] de Córdoba, S. F., "100 km. Altitude Boundary for Astronautics" [online article], http://www.fai.org/astronautics/100km.asp [retrieved Aug. 2007].

[3] Bird, G. A., Molecular Gas Dynamics and the Direct Simulation of Gas Flows, Clarendon Press, Oxford, 1994.

[4] Bird, G. A., "Forty Years of DSMC, and Now?," Proceedings of the 22nd International Symposium on Rarefied Gas Dynamics, American Inst. of Physics, Melville, NY, 2001, pp. 372-380.

[5] Dietrich, S., and Boyd, I. D., "Scalar and Parallel Optimized
Implementation of the Direct Simulation Monte Carlo Method," Journal of Computational Physics, Vol. 126, No. 2, 1996, pp. 328-342. doi:10.1006/jcph.1996.0141

[6] Maxwell, J. C., "On Stresses in Rarefied Gases Arising from Inequalities of Temperature," Philosophical Transactions of the Royal Society of London, Vol. 170, 1879, pp. 231-256. doi: $10.1098 /$ rstl.1879.0067

[7] Lord, R. G., "Application of the Cercignani-Lampis Scattering Kernel to Direct Simulation Monte Carlo Calculations," Proceedings of the 17th International Symposium on Rarefied Gas Dynamics, VCH, Weinheim, Germany, 1991, pp. 1427-1433.

[8] Millikan, R. A., "Coefficients of Slip in Gases and the Law of Reflection of Molecules from the Surfaces of Solids and Liquids," Physical Review, Vol. 21, No. 3, 1923, pp. 217-238. doi:10.1103/PhysRev.21.217

[9] Schamberg, R., "A New Analytic Representation of Surface Interaction for Hyperthermal Free-Molecule Flow with Application to Satellite Drag," Proceedings of the Heat Transfer and Fluid Mechanics Institute, Stanford Univ. Press, Palo Alto, CA, 1959, pp. 1-14.

[10] Nocilla, S., "On the Interaction between Stream and Body in FreeMolecule Flow," Proceedings of the 2nd International Symposium on Rarefied Gas Dynamics, Academic Press, New York, 1961, pp. 169208.

[11] Nocilla, S., "The Surface Re-Emission Law in Free Molecule Flow," Proceedings of the 3rd International Symposium on Rarefied Gas Dynamics, Vol. 2, Academic Press, New York, 1963, pp. 327-346.

[12] Hurlbut, F. C., and Sherman, F. S., "Application of the Nocilla Wall Reflection Model to Free-Molecule Kinetic Theory," Physics of Fluids, Vol. 11, No. 3, 1968, pp. 486-496. doi:10.1063/1.1691943

[13] Hurlbut, F. C., "Two Contrasting Modes for the Description of WallGas Interactions," Progress in Astronautics and Aeronautics, Vol. 158, AIAA, Washington, D.C., 1992, pp. 494-506.

[14] Schaaf, S. A., "Mechanics of Rarefied Gases," Encyclopedia of Physics: Fluid Dynamics 2, Vol. 8, Springer-Verlag, Berlin, 1963, pp. 591-624.

[15] Hinchen, J. J., and Foley, W. M., "Scattering of Molecular Beams by Metallic Surfaces," Proceedings of the 4th International Symposium on Rarefied Gas Dynamics, Vol. 2, Academic Press, New York, 1966, pp. 505-517.

[16] Knechtel, E. D., and Pitts, W. C., "Experimental Momentum Accommodation on Metal Surfaces of Ions Near and Above EarthSatellite Speeds," Proceedings of the 6th International Symposium on Rarefied Gas Dynamics, Vol. 2, Academic Press, New York, 1969, pp. 1257-1278.

[17] Kuščer, I., "Reciprocity in Scattering of Gas Molecules by Surfaces," Surface Science, Vol. 25, No. 2, 1971, pp. 225-237. doi:10.1016/0039-6028(71)90244-5

[18] Wenaas, E. P., "Equilibrium Cosine Law and Scattering Symmetry at the Gas-Surface Interface," Journal of Chemical Physics, Vol. 54, No. 1, 1971, pp. 376-388. doi:10.1063/1.1674619

[19] Cercignani, C., "Boundary Value Problems in Linearized Kinetic Theory," SIAM-AMS Proceedings, Vol. 1, American Mathematical Society, Providence, RI, 1969, pp. 249-268.

[20] Cercignani, C., and Lampis, M., "Kinetic Models for Gas-Surface Interactions," Transport Theory and Statistical Physics, Vol. 1, No. 2, 1971, pp. 101-114. doi: $10.1080 / 00411457108231440$

[21] Cercignani, C., and Frezzotti, A., "Numerical Simulations of Supersonic Rarefied Gas Flows Past a Flat Plate: Effects of the GasSurface Interaction Model on the Flowfield," Progress in Aeronautics and Astronautics, AIAA, Washington, D.C., Vol. 118, 1989, pp. 552566.

[22] Sharipov, F., "Application of the Cercignani-Lampis Scattering Kernel to Channel Gas Flows," Proceedings of the 22nd International Symposium on Rarefied Gas Dynamics, American Inst. of Physics, Melville, NY, 2001, pp. 347-353.

[23] Cercignani, C., Lampis, M., and Lentati, A., "A New Scattering Kernel in Kinetic Theory of Gases," Transport Theory and Statistical Physics, Vol. 24, No. 9, 1995, pp. 1319-1336. doi:10.1080/00411459508206026

[24] Cercignani, C., and Lampis, M., "New Scattering Kernel for GasSurface Interaction,” AIAA Journal, Vol. 35, No. 6, 1997, pp. 10001011. doi: $10.2514 / 2.209$

[25] Lord, R. G., "Some Extensions to the Cercignani-Lampis Gas-Surface Scattering Kernel," Physics of Fluids A: Fluid Dynamics, Vol. 3, No. 4, 
1991, pp. 706-710.

doi: $10.1063 / 1.858076$

[26] Lord, R. G., "Some Further Extensions of the Cercignani-Lampis GasSurface Interaction Model," Physics of Fluids, Vol. 7, No. 5, 1995, pp. 1159-1161. doi:10.1063/1.868557

[27] Ketsdever, A. D., and Muntz, E. P., "Gas-Surface Interaction Model Influence on Predicted Performance of Microelectromechanical System Resistojet," Journal of Thermophysics and Heat Transfer, Vol. 15, No. 3, 2001, pp. 302-307. doi: $10.2514 / 2.6626$

[28] Santos, W. F. N., "Influence of Gas-Surface Interaction on Hypersonic Aerothermodynamic Performance of Flat-Nose Power-Law Bodies," AIAA Paper 2006-1194, 2006.

[29] Utah, S., and Arai, H., "Monte Carlo Simulation of Reentry Flows Based Upon a Three-Temperature Model," Proceedings of the 23rd International Symposium on Space Technology and Science, Vol. 1, Japan Society for Aeronautical and Space Sciences, Tokyo, 2002, pp. 1209-1214.
[30] Schaaf, S. A., and Chambré, P. L., Flow of Rarefied Gases, Princeton Univ. Press, Princeton, NJ, 1961.

[31] Gombosi, T. I., Gaskinetic Theory, Cambridge Univ. Press, Cambridge, England, U.K., 1994.

[32] Cercignani, C., "Scattering Kernels for Gas-Surface Interactions," Transport Theory and Statistical Physics, Vol. 2, No. 1, 1972, pp. 27-53. doi:10.1080/00411457208231462

[33] Cecil, D. E., and McDaniel, J. C., "Planar Velocity and Temperature Measurements in Rarefied Hypersonic Flow Using Iodine LIF," AIAA Paper 2005-4695, 2005.

[34] HyperMesh., Software Package, Ver. 7.0, Altair Engineering, Inc., Troy, MI, 2004.

[35] Lide, D. R., CRC Handbook of Chemistry and Physics, 87th ed, Taylor and Francis, Boca Raton, FL, 2006.

[36] Cook, S. R., Hoffbauer M. A., and Cross, J. B., "Generating Velocity and Angular Distribution Functions of Molecules Scattered Off of Surfaces from Measurements of Forces Exerted on Surfaces by Incident Gases,” AIAA Paper 96-2227, 1996. 